\title{
An Audio Blind Watermarking Algorithm by Modifying the DWT Low-frequency Coefficients
}

\author{
Xuesong Chen ${ }^{1}$ Haotian $\mathrm{Li}^{1}$ Guanglong $\mathrm{Bu}^{1}$ Hongbo $\mathrm{Bi}^{1}$
}

\begin{abstract}
An audio watermarking algorithm by modifying the low-frequency coefficients of DWT is discussed in this paper. Audio signal is divided into many sections, whose quantity is equal to the size of the watermark. The maximal value and the second maximal value of DWT low frequency coefficient can be found in each section. Then we can extract the last non-zero number of the two extreme values. The watermark is embedded based on the two numbers' parity. We take the encryption twice in order to improve security of the watermark. Experiments show that after some common attacks such as resample, Gauss noise, requantization and low-pass filtering, the algorithm has good imperceptibility and robustness.
\end{abstract}

Keywords: Audio blind watermarking. DWT. Security

\subsection{Introduction}

Discrete wavelet transform ( $D W T$ ) has many advantages such as multi-scale and multi-resolution. It is good at processing one-dimensional signal, so it is widely used in the field of digital watermarking. Xu Dacheng proposed a non-blind audio watermarking algorithm basing on DWT. ${ }^{2}$ It can't realize blind extraction. Chen Licong proposed a blind audio watermark algorithm based on local extreme points. ${ }^{1}$ The algorithm is essentially to find the extreme value point of the wavelet low frequency coefficient. Watermark embedding is implemented by modifying the amplitude value of extreme points. Because the algorithm is complicated and needs large amount of calculation, so it is very difficult to realize. Zhang Zhijie ${ }^{3}$ proposed an algorithm by modifying intermediate frequency coefficients in wavelet domain. But comparing with the wavelet low frequency domain and intermediate frequency domain, low frequency domain usually has better robustness.

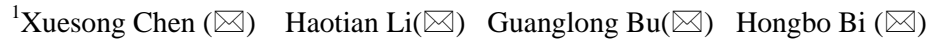

Electrical \& Information Engineering College, Northeast Petroleum University. 163318,

Daqing, China

e-mail: cxsnepu@163.com lihaotian0010@163.com
} 


\subsection{Watermark Image Preprocessing}

In order to compare the original watermark and the extracted watermark more intuitively, the watermark image uses the binary image which is named as $w_{0}$, and which size is $32 * 32$. Watermark sequence is encrypted twice for improving watermark's security. Fig. 1.1 is the watermark encryption procession.

Arnold scrambling is the first step. Then watermark image is downdimensionality denoted by $A$. Using the former 1024 numbers of the logistic chaotic sequence, the $X O R$ operation is made. The secondary encryption watermark is implemented. Fig. 1.2 is Encrypted watermark. And $w_{0}$ is original watermark in Fig. 1.2(a), $w_{1}$ is the first encrypted watermark by Arnold scrambling in Fig. 1.2(b), and $w_{2}$ is the second encrypted watermark by logistic chaotic encryption in Fig. 1.2(c).

\subsection{Algorithm Implementation}

\subsubsection{Watermark Embedding}

Because high frequency coefficients of wavelet describe the detail component of the audio signal, and low frequency coefficients describe the rough component of the audio signal, low frequency domain usually has better robustness besides comparing with low frequency domain and intermediate frequency domain. So we choose the low-frequency component of wavelet to embed the watermark. ${ }^{4}$ The information of encrypted watermark is embedded into the low frequency coefficients of wavelet in $D W T$ domain. Finally, the watermarked audio signal can be obtained by wavelet reconstructing, which is shown as Fig. 1.3.

\begin{tabular}{|c|c|c|c|}
\hline Original \\
Watermark
\end{tabular}$\rightarrow \begin{gathered}\text { Arnold } \\
\text { Scrambling }\end{gathered} \rightarrow \begin{gathered}\text { Logistic Chaotic } \\
\text { Encryption }\end{gathered} \rightarrow \begin{gathered}\text { Encrypted } \\
\text { Watermark }\end{gathered}$

Fig. 1.2 Watermark encryption procession
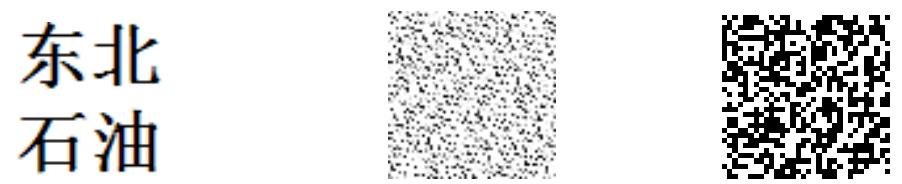

(a) Original watermark $w_{0}$ (b) Arnold scrambling $w_{1}$ (c) Logistic Chaotic Encryption $w_{2}$

Fig. 1.2 Encrypted watermark 


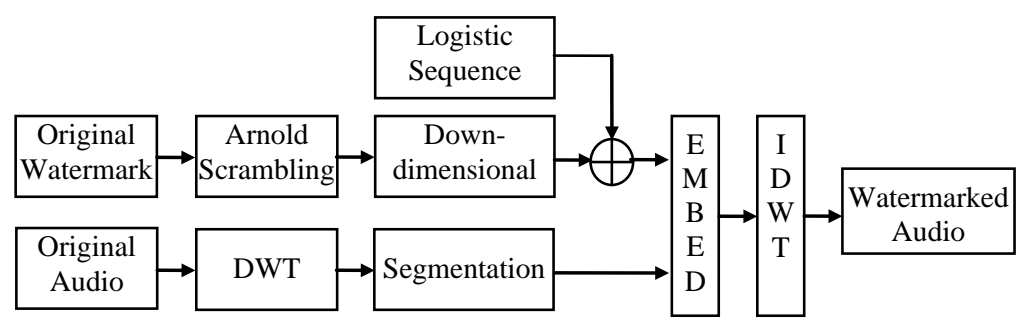

Fig. 1.3 Watermark Embedding Procession

1. Audio signal is divided into many sections. The quantity is equal to the size of the watermark $\left(M_{1} \times M_{2}\right)$. Each data segment has $\mathrm{N}$ sampling points. Fig. 1.4 is original audio segmentation schematic

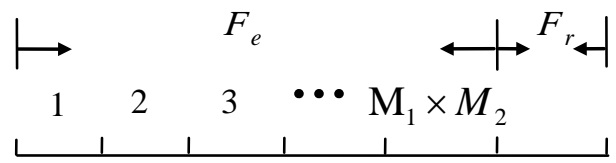

Fig. 1.4 Original audio segmentation schematic

Audio signal is divided into two sections.

$$
\mathrm{F}=F_{e}+F_{r}
$$

The relevant part of embedding watermark can be seen as $F_{e}$, whose size is $N \times M_{1} \times M_{2}$. This algorithm chooses the value of $N$ carefully. If the value of $N$ is too small, the proportion of $F_{e}$ is small over the entire audio and $F_{e}$ is clipped easily. Not all information is embedded if the value of $N$ is not too large. And $F_{r}$ is the independent part of embedding watermark. This part remains unchanged in the procession of watermark embedding.

2. L level discrete wavelet transforms may be done to the each data segment of $F_{e}$. We find the absolute value of the maximum value and the second maximum value from each L-level low-frequency component, which is denoted by $M A X 1$ and $M A X 2$ respectively. The last nonzero number of $M A X 1$ and $M A X 2$ is extracted and denoted by $M A X 1_{l}$ and $M A X 2_{l}$ respectively.

3. When the embedded watermark is "1", if $M A X 1_{1}$ mod 2 is equal to $M A X 2_{l}$ mod 2, you do not need to modify the value of $M A X 1_{1}$; if $M A X 1_{1}$ mod 2 is not equal to $M A X 2_{1}$ mod 2, you need to modify the value of $M A X 1_{1}$. If the value of $M A X 1_{1}$ is equal to 9, the value of $M A X 1_{l}^{\prime}$ is changed to $M A X 1_{l}-1$, the value of $M A X 1_{l}^{\prime}$ is equal to $M A X 1_{1}+1$ in other conditions. When the embedded watermark is " 0 ", if $M A X 1_{l} \bmod 2$ is not equal to $M A X 2_{l} \bmod 2$, you don't need to modify the value of $M A X 1_{1}$, if $M A X 1_{1}$ mod 2 is equal to $M A X 2_{l}$ mod 2; you need to modify the value of $M A X 1_{1}$. The rules are the same as you embed "1". 
4. After wavelet coefficients are adjusted, the wavelet reconstruction is needed to do. The new watermarked audio signal is completed.

\subsubsection{Watermark Extracting}

Fig. 1.5 is the procession of the watermark extracting. The fragmentation procession is added to the detected audio signal. $L$ level discrete wavelet transform is taken for each data segment of the part Fe. We find the absolute value of the maximum value and the second maximum value from each L-level low-frequency component, and the last nonzero number of which is extracted. If $M A X 1_{1}$ mod 2 is equal to $M A X 2_{1}$ mod 2, the extracted watermark information is "1"; otherwise the extracted watermark information is "0". A binary disorder watermark sequence can be obtained through these processions.

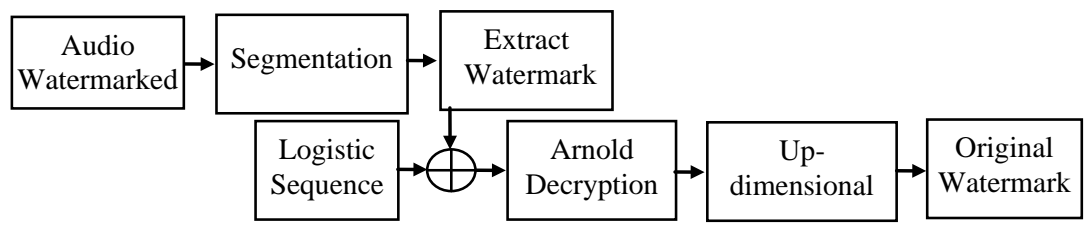

Fig. 1.5 Watermark extracting process

The logistic chaotic sequence is implemented by the key $K \quad\left(u=4, x_{0}=0.8\right)$ and select the former 1024 data. A series of binary disordered watermark sequence is obtained by taking $X O R$ procession. The first decrypted one-dimensional watermark signal is made. Up-dimensional procession is added to this onedimensional watermark signal and the two-dimensional watermark is obtained. The watermark, which we need, can be obtained.

\subsection{Results and Analysis of Experiments}

Two kinds of audio are used in the simulation. Its format is .wav, length is 5 seconds, sampling frequency is $22.05 \mathrm{kHz}$, and resolution is 16 bit. Watermark image is binary gray-scale image which size is $32 * 32$. $\mathrm{Db} 4$ wavelet is used for threelevel wavelet decomposition.

Imperceptibility and robustness is mainly tested. By hearing tests, the audio signals have no significant difference with embedded watermark.

Fig. 1.6 and Fig. 1.7 are pop and rock music with the watermarked audio waveform and coefficients. Watermarked audio signal is attacked by following attacks in robustness tests. 
1. White Gaussian noise: Gaussian noise is normal distribution function, with 0.01 of expected value, 0.05 of variance, and it noise contains with embedded audio signals information;

2. Colored noise: Colored noise is achieved by a cut-off frequency of the low-pass filter of $10 \mathrm{kHz}$. It is generated and added to embedded audio signals information;

3. Re-quantization: 16 bit audio signal is quantized to 8 bit firstly, then is quantized to 16 bit;

4. Re-sampling: Up-sampling: Audio signal sampling frequency is become from $22.05 \mathrm{kHz}$ to $44.1 \mathrm{kHz}$, then using extraction technology restore to $22.05 \mathrm{kHz}$ of the original sampling frequency. Down-sampling: Audio signal sampling frequency is become from $22.05 \mathrm{kHz}$ to $11.025 \mathrm{kHz}$, then using extraction technology restore to $22.05 \mathrm{kHz}$ of the original sampling frequency.

5. Random cut: 10 positions are selected randomly from the embedded audio signals. 200 samples are cut at these positions. Then we take watermark extraction procedure.

6. Low-pass filtering: Butterworth low-pass filter's order is 6; and its cutoff frequency is $10 \mathrm{kHz}$. Low-pass filtering operation is taken for the audio signal with the watermark information.

Fig. 1.6 and Fig. 1.7 show that audios have small changes with watermark embedded. Human ear is difficult to detect this different, and this algorithm has better imperceptibility.

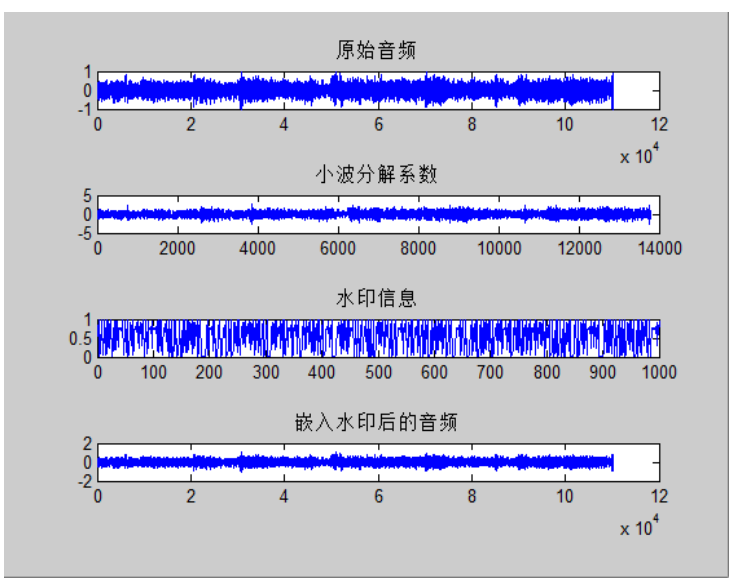

Fig. 1.6 Pop waveform comparison chart 


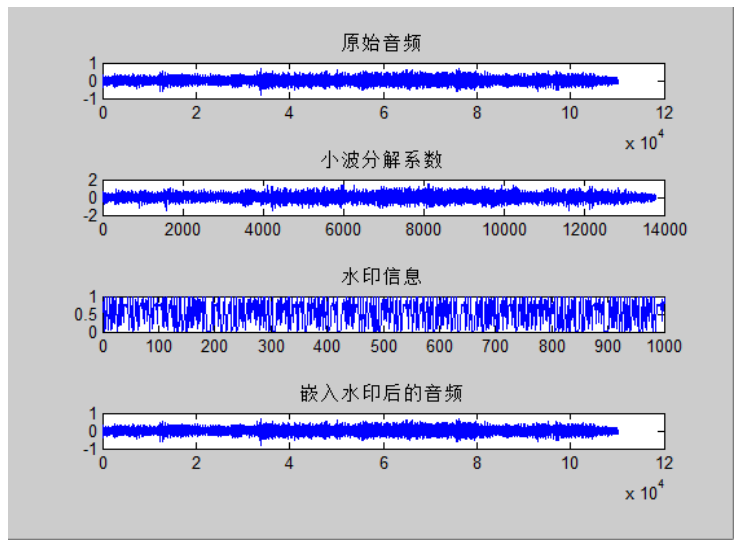

Fig. 1.7 Rock music waveform comparison chart

Table 1.1 Extracted watermark after various attacks

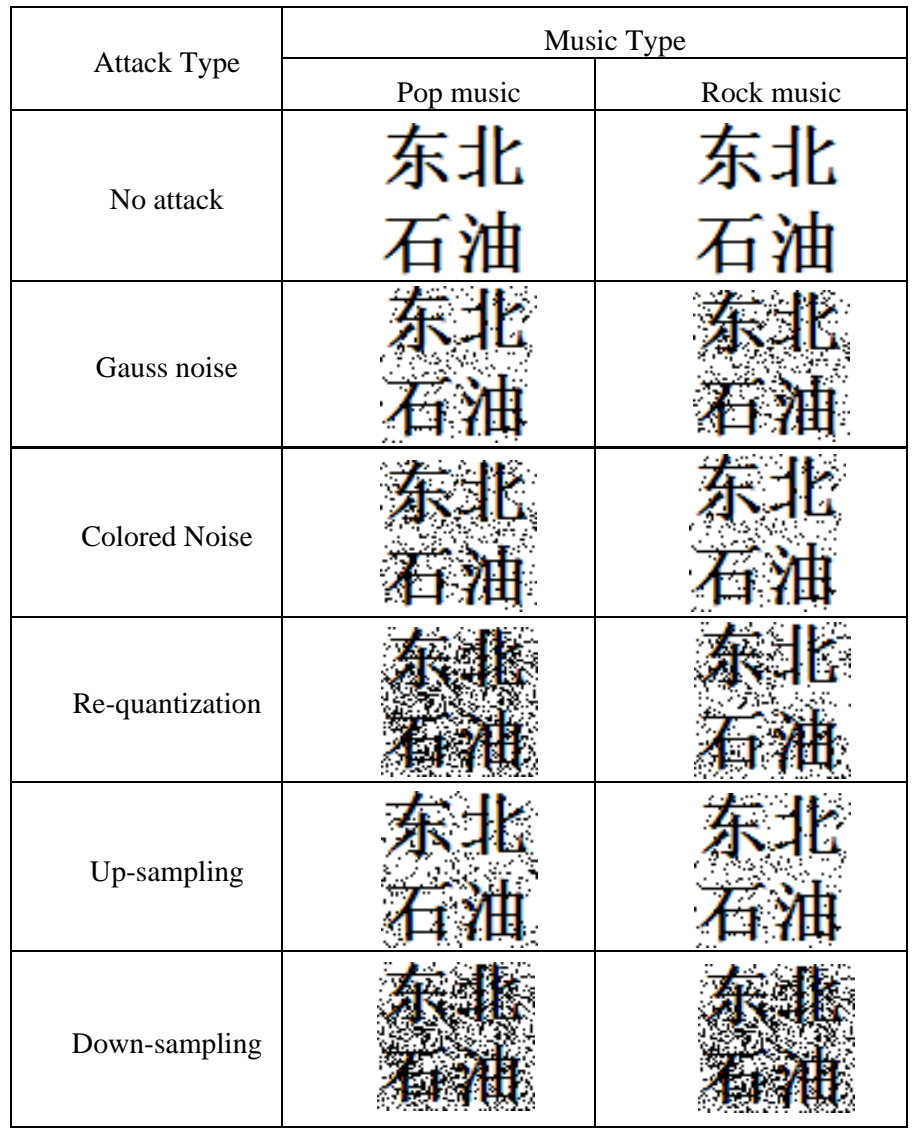


Table 1.1 (continued)

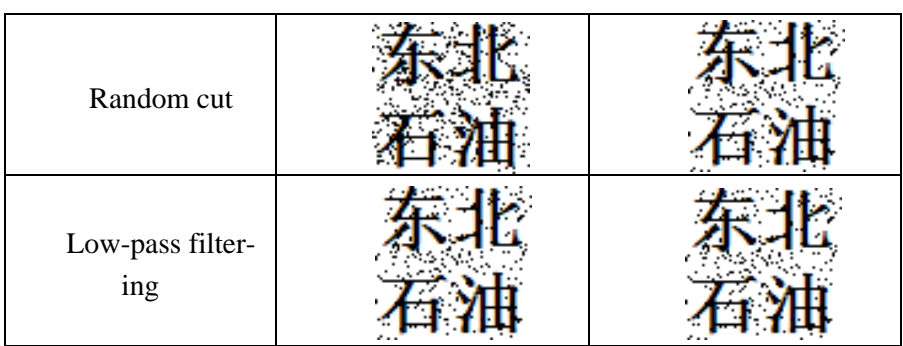

Table 1.2 Watermark correlation coefficient and contrast

\begin{tabular}{|c|c|c|c|c|}
\hline \multirow{2}{*}{ Attack Type } & \multicolumn{4}{|c|}{ Music Type } \\
\cline { 2 - 5 } & \multicolumn{2}{|c|}{ Pop music } & \multicolumn{2}{c|}{ Rock music } \\
\cline { 2 - 5 } & $N C$ & $S N R$ & $N C$ & $S N R$ \\
\hline No attack & 1.0000 & 34.45 & 1.0000 & 34.57 \\
\hline Gauss noise & 0.8796 & 24.86 & 0.8814 & 25.03 \\
\hline Colored Noise & 0.9035 & 24.57 & 0.9057 & 24.96 \\
\hline Re-quantization & 0.7826 & 21.54 & 0.7819 & 21.68 \\
\hline Up-sampling & 0.8568 & 27.66 & 0.8525 & 26.98 \\
\hline Down-sampling & 0.7457 & 23.68 & 0.7521 & 24.81 \\
\hline Random cut & 0.8515 & 21.79 & 0.8512 & 22.06 \\
\hline Low-pass filtering & 0.9223 & 20.17 & 0.9247 & 20.45 \\
\hline
\end{tabular}

The correlation coefficient and the signal to noise ratio is from Table 1.1 and Table 1.2. The watermarked audio is attacked by common attacks; although the clarity of the extracted watermark is different, but they can still be identified, and that this algorithm has better robustness.

\subsection{Conclusions}

A blind audio watermarking algorithm by modifying the low-frequency coefficients of $D W T$ is proposed. The watermark takes secondary encryption process, which is to improve the security of watermark. The algorithm is simple and fast. Simulation results show that audios have small changes with watermark embedded. Human ear is difficult to detect the different. The watermarked audio is attacked by common attacks; and the watermark extracted can be identified. It proves that this algorithm has better robustness. If the keys are not correct, the watermark information is not extracted correctly. The algorithm's imperceptibility is good, but its robustness is relatively poor. How to balance the imperceptibility and robustness is the direction of our future research work. 
This work is supported by the Science \& Technology Project of Heilongjiang province under Grant No. 12521056.

\subsection{References}

1. Chen Licong, Yao Zhiqiang (2005) Based on Local Extreme Points of the Audio Watermarking Algorithm. Computer Engineering and Applications, 41 (19): 41-43

2. Xu Dacheng, Shi Minghui (2002) DWT-based Digital Audio Watermarking Technology Research. In: Communications Technology, (11): 86-87

3. Zhang Zhijie, Ji Qichun, Wang Huiqin (2004) A New Digital Audio Watermarking Algorithm . Xi'an University of Architecture and Technology, (2): 203-206

4. Zhu Youzhong, Xiang Jun (2010) A Blind Audio Watermarking Algorithm Based on DWT and Relatively Small Values. In: Journal of Jilin University, (2): 203-208 